\title{
Common Categories and Data for Application Interoperability
}

\author{
Kalaiselvi Arunachalam \\ Research Scholar, School of Computer Science, \\ Engineering and Applications, Bharathidasan University, \\ Tiruchirappalli, India.
}

\author{
Dr. Gopinath Ganapathy \\ Registrar, Bharathidasan University, \\ Tiruchirappalli, India.
}

\begin{abstract}
Millions of applications from several categories of application marketplaces are used by billions of users on their devices like Smartphone, Tablet, Phablet, Smart TV, Notebook etc. around the world. These applications serve the users on different segments like education, health, finance, business, entertainment, shopping etc. The sharing of data between these applications would be more beneficial to the users when they use these diverse devices together in their home network. This paper presents all categories of applications available on popular application marketplaces and the common data types that can be shared across these applications in a home network using UPnP in which application interoperability is achieved by extending the UPnP Device Architecture. This paper also discusses about the common data formats, data transfer formats, data communication medium and data flow which can be used to achieve communication between these diverse applications in a home network.
\end{abstract}

Keywords: Application Data, Application Categories, Application Marketplace, Home Network, UPnP.

\section{INTRODUCTION}

There are millions of applications from different segments like education, health, finance, business, entertainment, shopping etc. are used by billions of users on their devices like Smartphone, Tablet, Phablet, Smart TV, Notebook etc. These applications provide different types of data like text, URL, audio, video, picture etc. to the users for their daily requirements. These diverse data would be required across applications in order to provide effective communication and production at a minimal time to the user when these heterogeneous devices are used together in a home network. These data can be shared across applications on heterogeneous devices which are connected together through various network medium like $\mathrm{Wi}-\mathrm{Fi}$, Ethernet, Bluetooth etc. and the data transfer rate depends upon the type of underlying network medium in a home network. This paper presents all application categories from the popular application marketplaces and identifies the common data types that can be used to share data across these applications in a home network using UPnP in which application interoperability is achieved by extending the UPnP Device Architecture. Also, this paper discusses about the common data formats, data transfer formats, data communication medium and data flow which can be used to achieve communication between these diverse applications in a home network.

\section{PLATFORMS AND APPLICATIONS}

The devices ranging from a smart key chain to a smart car are available from various manufacturers with different operating systems like Android, iOS, BlackBerry OS, Windows 10 Mobile, Ubuntu Touch, Tizen, Firefox OS etc. Millions of applications are available on the application marketplaces or online stores of these operating systems and thousands of applications are added to these online stores each day by the developers or companies around the world. These application marketplaces enable the users to browse, download and install applications of their interest from various categories on their devices either paid or free of cost. Some of the popular application marketplaces are App Store as in [1], Google Play as in [2], BlackBerry World as in [3], Windows Store as in [4], Opera Mobile Store as in [5], Ubuntu App Store as in [6], Amazon App Store as in [7] etc. These application marketplaces provide millions of applications as in [Table 1] [8] to their users worldwide.

Table 1. Popular Application Marketplaces vs. Applications

\begin{tabular}{|l|l|l|}
\hline $\begin{array}{l}\text { Application } \\
\text { Marketplace }\end{array}$ & Operating System & $\begin{array}{l}\text { Total Apps } \\
\text { as of 2018) }\end{array}$ \\
\hline App Store & iOS & $2,200,000$ \\
\hline Google Play & Android & $2,800,000$ \\
\hline $\begin{array}{l}\text { BlackBerry } \\
\text { World }\end{array}$ & BlackBerry OS & 234,500 \\
\hline Windows Store & Microsoft Windows & 669,000 \\
\hline $\begin{array}{l}\text { Opera Mobile } \\
\text { Store }\end{array}$ & $\begin{array}{l}\text { Android, iOS, } \\
\text { Windows, }\end{array}$ & $300,000^{\mathrm{a}}$ \\
\hline $\begin{array}{l}\text { Ubuntu App } \\
\text { Store }\end{array}$ & BlackBerry, Symbian & $45,000^{\mathrm{b}}$ \\
\hline $\begin{array}{l}\text { Amazon App } \\
\text { Store }\end{array}$ & Android, BlackBerry & 450,000 \\
\hline \multicolumn{2}{|l|}{ a.as of November 2014, ${ }^{\text {b.as of October } 2015}$} \\
\hline
\end{tabular}

The applications are downloaded from various categories on these stores by the users on their devices like Smartphone, Tablet, Notebook etc. The number of app downloads from these stores are increasing day by day in to billions now as in [Figure 1] [9] which would be a demand for application interoperability across devices in a very near future. 


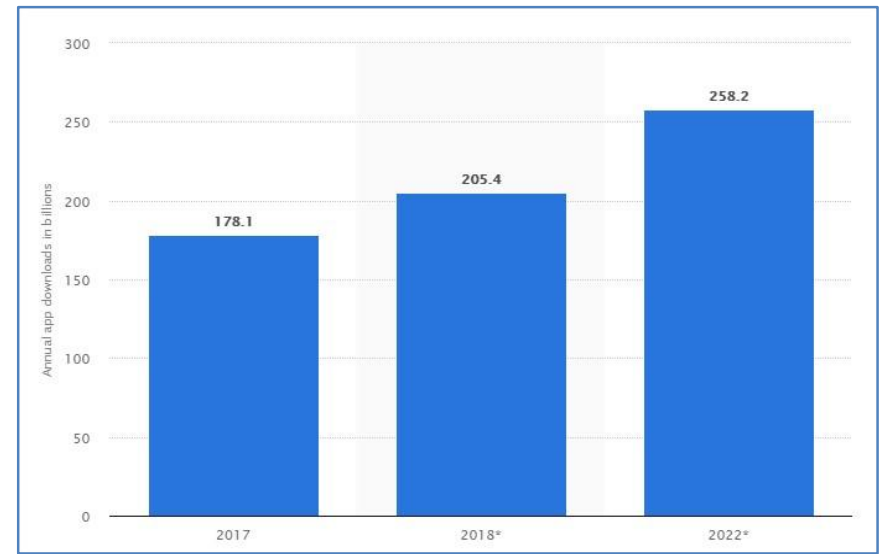

Fig. 1. Fig. 1. Number of app downloads worldwide in 2017, 2018 and $2022 *$ (estimated)

\section{APPLICATION CATEGORIES}

Millions of applications are available in the application marketplaces on various categories and the users are opted to browse, download and install them based on their interest. There are some common application categories which are available on all application marketplaces as in [Table 2]. The application marketplaces are organizing their apps based on the features provided by the app, device type and operating system of the device.

Table 2. Common Application Categories in Application Marketplaces

\begin{tabular}{|c|c|c|c|c|}
\hline $\begin{array}{l}\text { Application } \\
\text { Category }\end{array}$ & $\begin{array}{l}\text { App } \\
\text { Store }\end{array}$ & $\begin{array}{l}\text { Google } \\
\text { Play }\end{array}$ & $\begin{array}{l}\text { Windows } \\
\text { Store }\end{array}$ & $\begin{array}{l}\text { BlackBerry } \\
\text { World }\end{array}$ \\
\hline Books & $\Omega$ & $\checkmark$ & $\Omega$ & $\checkmark$ \\
\hline Business & $\checkmark$ & $\checkmark$ & $\checkmark$ & $\nearrow$ \\
\hline Education & $\checkmark$ & $\checkmark$ & $\checkmark$ & $\nearrow$ \\
\hline Entertainment & $\checkmark$ & $\checkmark$ & $\checkmark$ & 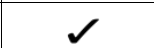 \\
\hline Finance & $\checkmark$ & $\checkmark$ & $\checkmark$ & $\Omega$ \\
\hline Games & $\mathscr{\nearrow}$ & $\checkmark$ & $\checkmark$ & $\mathcal{L}$ \\
\hline Health \& Fitness & $\checkmark$ & $\checkmark$ & 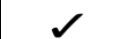 & $\mathcal{J}$ \\
\hline Lifestyle & $\mathcal{J}$ & $\mathcal{L}$ & $\mathcal{L}$ & 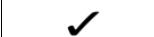 \\
\hline $\begin{array}{l}\text { Magazines \& } \\
\text { News }\end{array}$ & $\Omega$ & $\checkmark$ & $\checkmark$ & $\Omega$ \\
\hline $\begin{array}{l}\text { Maps \& } \\
\text { Navigation }\end{array}$ & $\mathscr{L}$ & $\mathscr{L}$ & $\mathscr{L}$ & $\mathscr{J}$ \\
\hline Music \& Audio & $\checkmark$ & $\mathscr{L}$ & 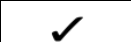 & $\checkmark$ \\
\hline Photo \& Video & 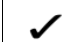 & $\checkmark$ & $\checkmark$ & $\Omega$ \\
\hline Productivity & $\Omega$ & $\Omega$ & 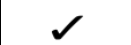 & 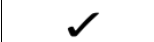 \\
\hline Shopping & $\checkmark$ & $\checkmark$ & $\checkmark$ & $\checkmark$ \\
\hline $\begin{array}{l}\text { Social } \\
\text { Networking }\end{array}$ & $\checkmark$ & $\checkmark$ & $\checkmark$ & $\checkmark$ \\
\hline Sports & $\checkmark$ & $\mathscr{L}$ & $\mathscr{L}$ & $\checkmark$ \\
\hline Travel & $\Omega$ & $\checkmark$ & $\checkmark$ & $\Omega$ \\
\hline Utilities & 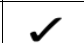 & $\checkmark$ & 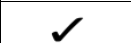 & $\checkmark$ \\
\hline
\end{tabular}

\section{APPLICATION DATA TYPE}

A data type is a classification that defines which type of value can be stored in a variable and what kind of mathematical, relational or logical operations can be performed on it. A String data type is used to store a text in a variable, a Float data type is used to store a decimal number in a variable etc. The data types can be classified in to basic data types, abstract data types and multimedia data types. These data types are used in various kinds of applications like desktop publishing, database, word processing, presentation, email, utility, enterprise, multimedia, spreadsheet, graphics, communication etc.

\section{A. Basic Data Types}

These are the basic building blocks of data manipulation in almost all programming languages towards the development of applications. Some of the basic data types are as follows.

- Integer - This data type is used to store whole numbers like 5, 100, 1525 etc.

- Float - This data type is used to store decimal numbers like 5.04, 3.52, 00.87 etc.

- Character - This data type is used to store single characters like 's', '5', "\#" etc.

- String - This data type is used to store text or alphanumeric characters like "My Research","a123" etc.

- Boolean - This data type is used to store logical values like "TRUE" and "FALSE".

- Date - This data type is used to store date information like "2017-05-27".

- Time - This data type is used to store time information like "15:36:42".

\section{B. Abstract Data Types}

These are the data types with an organized collection of information and a set of operations that are used to manage that information. Some of the abstract data types are as follows.

- List - Its a flexible structure that can grow or shrink on demand and the elements can be accessed, inserted, or deleted at any position within a list.

- Map - Its a kind of data structure that stores data in the form of key and value pairs where every key is unique.

- Queue - Its a kind of list where items are inserted at one end and deleted at the other end which is referred as "FIFO" (first-in-first-out) list.

- Tree - Its a kind of hierarchical structure on a collection of items and are used to represent organization charts, database systems etc.

- Stack - Its a kind of list where all insertions and deletions take place at one end called the Top which is referred as "LIFO" (last-in-first-out) list.

\section{Multimedia Data Types}

These data types includes the media data like text, images, audio, video, graphic objects etc. Some of the multimedia data types are as follows. 
International Journal of Applied Engineering Research ISSN 0973-4562 Volume 14, Number 6 (2019) pp. 1379-1383

(C) Research India Publications. https://dx.doi.org/10.37622/IJAER/14.6.2019.1379-1383

- Text - It is used to store text content.

- Image - It is used to store still images and the space occupied by the images are based upon the size, resolution, compression scheme etc.

- Audio - It is used to store audio content and the space occupied would be around 2 to $3 \mathrm{MBs}$ for an one minute of audio content.

- Video - It is used to store video content and the space occupied would be around $1 \mathrm{MB}$ for a single video frame.

- Graphic Object - It is used to define multimedia objects and to store 2D and 3D shapes.

\section{$\begin{array}{llll}\text { DATA TYPES FOR } & \text { APPLICATION }\end{array}$ INTEROPERABILITY}

Though several data types are used by various kinds of applications, there are some common data types which are universal across them regarding peer-to-peer communication across devices or applications. The popular operating systems like Android, iOS, Windows 10 Mobile and BlackBerry are using a few data types for application interoperability within their operating system or device. The Android mobile operating system uses the data types like string, URI and binary for application interoperability within a device as in [10]. The iOS mobile operating system uses string, URI and binary for application interoperability within a device and with other devices as well as in [11]. The BlackBerry mobile operating system uses string, URI and binary for application interoperability within a device as in [12]. The Windows 10 mobile operating system uses string, URI and binary data types for application interoperability within a device as in [13].

In a home network, the data types like integer, float, char, string, date, time, boolean, binary, URI etc. are used by UPnP for device interoperability as in [14].

\section{A. Common Data Types}

Based on the data types that are used in device interoperability and application interoperability as in [10]-[14], the common data types are identified as in [Table 3] in order to achieve application interoperability across devices in a home network.

Table 3. Common Data Types for Application Interoperability

\begin{tabular}{|l|l|}
\hline Data Type & Description \\
\hline Integer & Whole numbers (signed and unsigned) \\
\hline Float & 7 digits (32 bit) - Single Precision \\
\hline Double & $15-16$ digits (64 bit) - Double Precision \\
\hline Decimal & $28-29$ digits (128 bit) \\
\hline Character & Single character \\
\hline String & Multiple or alphanumeric characters \\
\hline
\end{tabular}

\begin{tabular}{|l|l|}
\hline Date & Date (DD/MM/YYYY) \\
\hline Time & Time (HH:MM:SS) \\
\hline Boolean & "1" for TRUE and "0" for FALSE \\
\hline BLOB & Binary Large Object (for Multimedia) \\
\hline UUID & Universally Unique IDentifier \\
\hline URI & Universal Resource Identifier \\
\hline
\end{tabular}

\section{$\begin{array}{llll}\text { DATA } & \text { FORMATS } & \text { FOR }\end{array}$ INTEROPERABILITY}

A data format is organized information based on a predefined specification so that the information can be stored or accessed or transmitted efficiently. The diverse applications are using a variety of data formats based on their category like an audio player application supports audio files with different audio formats. The popular data formats as in [Table 4] which can be used for application interoperability in order to share data between applications in a home network.

Table 4. Data Formats for Application Interoperability

\begin{tabular}{|l|l|}
\hline Data Type & Data Formats \\
\hline Text & $\begin{array}{l}\text { RTF, TXT, DOC, DOCX, HTML,XML, } \\
\text { PDF }\end{array}$ \\
\hline $\begin{array}{l}\text { Tabular } \\
\text { Data }\end{array}$ & CSV, XLS, XLSX, DBF, MDB, ODS, TXT \\
\hline Image & $\begin{array}{l}\text { JPEG, TIF, TIFF, RAW, PSD, PNG, GIF, } \\
\text { BMP }\end{array}$ \\
\hline Audio & $\begin{array}{l}\text { MP3, AIF, WAV, AMR, OGG, WMA, } \\
\text { M4P }\end{array}$ \\
\hline Video & MOV, AVI, MP4, FLV, WMV, 3GPP, RM \\
\hline $\begin{array}{l}\text { Graphics } \\
\text { SVG, PSD, SWF, CGM, XPM, WEBP }\end{array}$ \\
\hline $\begin{array}{l}\text { Geospatial } \\
\text { Data }\end{array}$ & $\begin{array}{l}\text { GeoTIFF, JPEG2000, DRG, GDF, ECW, } \\
\text { CIB }\end{array}$ \\
\hline
\end{tabular}

\section{DATA TRANSFER FORMATS FOR APPLICATION INTEROPERABILITY}

The XML [15] and JSON [16] are the two popular data transfer formats which are widely used already for data interchange across the web. Both of them are simple, open, humanreadable, machine-readable, inter-operable and with structured data that can be used by applications. JSON is faster than XML as because of its simple structure. 
The UPnP uses XML to provide device and service descriptions to the control points in order to communicate, control and share data between UPnP devices in a home network. Hence XML can be used for application interoperability in a home network. JSON can also be used for application interoperability based on the type of data, size of data, nature of application etc.

\section{DATA COMMUNICATION MEDIUM APPLICATION INTEROPERABILITY}

FOR

The communication medium involves the wired or wireless connections between electronic devices for the transmission of digital information across them in a network. The communication medium of different types like Ethernet, Wi-Fi, FireWire, Bluetooth, G.hn, IrDA etc. are already used in a home network for device interoperability as in [17]. The data transfer rate varies depends upon the communication medium used in the network as in [Table 5]. These communication medium can also be used for application interoperability for an effective data transfer across the diverse applications. Based on the communication medium chosen by a device in a network, an application shall also use the same as it resides on the device itself.

Table 5. Data Transfer Rate in Communication Medium

\begin{tabular}{|l|l|}
\hline Communication Medium & $\begin{array}{l}\text { Data Transfer } \\
\text { Rate }\end{array}$ \\
\hline Ethernet & $10 \mathrm{Mbit} / \mathrm{s}$ \\
\hline Fast Ethernet & $100 \mathrm{Mbit} / \mathrm{s}$ \\
\hline Gigabit Ethernet & $1 \mathrm{Gbit} / \mathrm{s}$ \\
\hline Wi-Fi (IEEE 802.11n) & $54 \mathrm{Mbit} / \mathrm{s}$ \\
\hline FireWire (IEEE 1394b) & $3145.7 \mathrm{Mbit} / \mathrm{s}$ \\
\hline Bluetooth (5.0) & $50 \mathrm{Mbit} / \mathrm{s}$ \\
\hline G.hn & $1 \mathrm{Gbit} / \mathrm{s}$ \\
\hline IrDA (UFIR) & $96 \mathrm{Mbit} / \mathrm{s}$ \\
\hline
\end{tabular}

\section{$\begin{array}{llll}\text { DATA } & \text { FLOW } & \text { FOR } & \text { APPLICATION }\end{array}$ INTEROPERABILITY IN A HOME NETWORK}

Based on the proposed UPnP Application Architecture as in [18], the application interoperability can be achieved in a home network and the data flow between the applications as in [Figure 2] occurs at all phases like discovery, advertisement, description, control, eventing and presentation whereby applications can discover, share data and control each other in a home network despite of their device type and operating system.

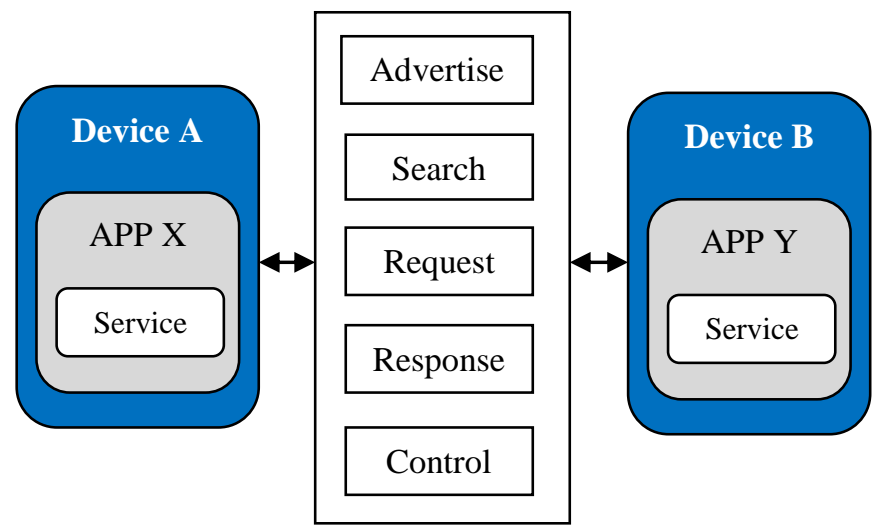

Fig. 2. Data flow between two UPnP applications in a home network

\section{CONCLUSION}

The wide array of smart devices and their massive presence around the world resulted with millions of applications from various categories in the application marketplaces for the users. The sharing of data between these applications would be beneficial to the users while using these diverse devices together in a home network. Based on this requirement, all categories of applications across the popular application marketplaces are analyzed and common categories are identified from them. The data types used by the applications on different platforms are analyzed and the common data types are identified from them. These common data types can be used in those common application categories to achieve application interoperability in a home network using UPnP. In addition to the data types, the data formats, data transfer formats, data communication medium and data flow which can be used for effective communication between applications are discussed.

\section{REFERENCES}

[1] App Store (iOS): https://apple.com/appstore

[2] Google Play: https://play.google.com/store

[3] BlackBerry World: http://appworld.blackberry.com/

[4] Microsoft Store: https://www.microsoft.com/store/apps

[5] Opera Mobile Store: http://apps.opera.com/

[6] Ubuntu App Store: https://apps.ubuntu.com/

[7] Amazon App Store: http://www.amazon.com/appstore

[8] App stores: number of apps in leading app stores 2017 Statista: https://www.statista.com/statistics/276623/ number-of-apps-available-in-leading-app-stores/

[9] Annual number of global mobile app downloads 2022/Statista: https://www.statista.com/statistics /271644/worldwide-free-and-paid-mobile-app-storedownloads/ 
International Journal of Applied Engineering Research ISSN 0973-4562 Volume 14, Number 6 (2019) pp. 1379-1383

(C) Research India Publications. https://dx.doi.org/10.37622/IJAER/14.6.2019.1379-1383

[10] Sending the User to Another App:

https://developer.android.com/training/basics/intents/sen ding.html

[11] Inter-App Communication:

https://developer.apple.com/library/content/documentatio n/iPhone/Conceptual/iPhoneOSProgrammingGuide/Inter -AppCommunication/Inter-AppCommunication.html

[12] Invocation framework - BlackBerry Native: https://developer.blackberry.com/native/documentation/d evice_platform/invocation/invocation_framework.html

[13] App-to-app communication - UWP app developer | Microsoft Docs: https://docs.microsoft.com/enus/windows/uwp/app-to-app/

[14] Andrew D., Bryan R., Maarten B., John G., Alan M., YoonSoo K., Bruce F., and Jonathan T., 2015, "UPnP Device Architecture 2.0", Open Connectivity Foundation (OCF), United States.
[15] Extensible Markup Language (XML) 1.0 (Second Edition), (2000) The World Wide Web Consortium (W3C).

[16] The JavaScript Object Notation (JSON) Data Interchange Format (RFC7159), (2014) The Internet Engineering Task Force (IETF).

[17] Oliver K., and Shin S., 2014, "System and method for improving service and device discovery in a UPnP-based wireless communication network", US Patent, United States.

[18] Kalaiselvi A., and Gopinath G., 2017, "Extending UPnP for Application Interoperability in a Home Network", International Journal of Electrical and Computer Engineering (IJECE), Vol. 7, No. 4, pp. 2085-2093. 